\title{
EXTENSION ET DIVISION DANS LES VARIÉTÉS À CROISEMENTS NORMAUX
}

\author{
Abderrabi Maati et Emmanuel Mazzilli
}

\begin{abstract}
Let $D$ be a bounded strictly pseudoconvex domain with smooth boundary and $f=\left(f_{1}, \ldots, f_{p}\right)\left(f_{i} \in \operatorname{Hol}(\bar{D})\right)$ a complete intersection with normal crossing. In this paper we study an extension problem in $L^{\infty}$-norm for holomorphic functions defined on $f^{-1}(0) \cap D$ and a decomposition formula $g=\sum_{i=1}^{p} f_{i} g_{i}$ for holomorphic functions $g \in I_{\left(f_{1}, \ldots, f_{p}\right)}(D)$ in Lipschitz spaces. We stress that for the two problems the classical theorem cannot be applied because $f^{-1}(0)$ has singularities on the boundary $\partial D$. This work is the first step to understand this type of problem in the general singular case.
\end{abstract}

\section{Introduction}

De nombreux auteurs ont étudié les idéaux de fonctions holomorphes avec des conditions de croissance. Plus précisément, considérons l'idéal engendré par $\left(f_{1}, \ldots, f_{p}\right), p$ fonctions holomorphes définies au voisinage d'un domaine $D$ strictement pseudoconvexe, que l'on note $I_{f_{1}, \ldots, f_{p}}$. On s'intéresse au problème suivant: étant donné une fonction, $f$, de $I_{f_{1}, \ldots, f_{p}} \cap L^{p}(D)$, peut-on trouver une décomposition de $f$ dans $\left(f_{1}, \ldots, f_{p}\right)$ avec des coefficients dans $L^{p}(D)$ (sinon dans quels $L^{q}$ peut-on trouver des coefficients?)? L'un des plus fameux résultats dans ce sens est le Théorème de division de Skoda (voir [15]) qui donne, en schématisant, une condition analytique suffisante pour que l'on ait une décomposition dans $L^{2}$ (il est à noter que le domaine est pseudoconvexe sans aucune restriction). Malheureusement, cette condition n'est nécessaire que si $\left(f_{1}, \ldots, f_{p}\right)$ sont des coordonnées locales en tous les points de $\left\{f_{1}=\cdots=f_{p}=0\right\}$. Dans cet article, nous étudions des problèmes de division, dans les espaces de Lipschitz, avec $\left(f_{1}, \ldots, f_{p}\right)$ qui définie une intersection compléte à croisements normaux (voir cidessous pour les définitions); on peut noter que dans ([3]), les auteurs

2000 Mathematics Subject Classification. 32A25, 32A27.

Mots-clés. Courants résiduels, domaines strictement pseudoconvexes, représentations intégrales, croisements normaux. 
ont obtenu des résultats de décomposition dans les espaces de Lipschitz pour les fonctions holomorphes, en supposant que $\left(f_{1}, \ldots, f_{p}\right)$ définissait des coordonnées locales en tous points de $\left\{f_{1}=\cdots=f_{p}=0\right\}$. On étudie également un problème d'extension de fonctions holomorphes définies sur une sous-variété à croisements normaux d'un domaine strictement pseudoconvexe. Beaucoup de résultats dans cette direction sont connus si l'on suppose que la sous-variété est sans singularité près du bord, c'est pourquoi l'étude dans les croisements normaux semble être le premier pas vers le cas singulier (on peut ajouter que le cas singulier se ramène toujours au cas à croisements normaux par morphisme d'Hironaka).

\section{Définitions et notations}

On considére $D \Subset D^{\prime}$, où $D^{\prime}$ est un domaine relativement compact de $\mathbb{C}^{n}$.

Définition 0.1. Soit $f: D^{\prime} \rightarrow \mathbb{C}^{p}, z \rightarrow\left(f_{1}(z), \ldots, f_{p}(z)\right)$ une application holomorphe; on dit que $f$ est une intersection complète sur $D$ si et seulement si $f^{-1}(0)$ est de dimension pure $n-p$ dans $D(1 \leq p \leq n-1)$.

Définition 0.2. Soit $f: D^{\prime} \rightarrow \mathbb{C}^{p}$ une intersection complète sur $D$; on dit que $f$ est à croisements normaux sur $\partial D$ si et seulement si $\forall z^{0} \in f^{-1}(0) \cap \partial D$, il existe un changement de coordonnées holomorphes (local), tel que:

$$
\forall i \in\{1, \ldots, p\}, \quad f_{i}(z)=h_{i}(z)\left(z_{1}-z_{1}^{0}\right)^{\alpha_{i, 1}} \times \cdots \times\left(z_{n}-z_{n}^{0}\right)^{\alpha_{i, n}},
$$

avec $h_{i}$ une unité.

Soit $f=\left(f_{1}, \ldots, f_{p}\right)$ une intersection complète à croisements normaux. Pour $z_{0} \in \partial D \cap\left\{z \in D^{\prime} / f_{1}(z)=\cdots=f_{p}(z)=0\right\}$, on considère

$$
f_{i}=u_{i, 1, z_{0}}^{\alpha_{i, 1, z_{0}}} \times \cdots \times u_{i, p_{i}, z_{0}}^{\alpha_{i, p_{i}, z_{0}}}
$$

la décomposition en facteurs irréductibles de $f_{i}$ au point $z_{0}$. Il est à noter que l'hypothèse $f$ à croisements normaux entraîne que $p_{i} \leq n$ et $u_{i, k, z_{0}}$ sont des coordonnées locales près de $z_{0}$.

Définition 0.3. Soit $f: D^{\prime} \rightarrow \mathbb{C}^{p}$ une intersection complète à croisements normaux sur $\partial D$; on dit que $f$ est à croisements normaux transverses à $\partial D$, si et seulement si $\forall z \in \partial D$ :

$$
\partial \rho(z) \notin \sum_{j} \operatorname{Span}_{\mathbb{C}}\left\langle\partial u_{j, 1, z}(z), \ldots, \partial u_{j, p_{j}, z}(z)\right\rangle,
$$

où $D=\{\rho<0\}$. 
Remarque. Dans le cas régulier, cette définition s'écrit de la manière habituelle:

$$
\partial \rho(z) \wedge \partial f_{1}(z) \wedge \cdots \wedge \partial f_{p}(z) \neq 0
$$

Définition 0.4. Soit $V$ un sous-ensemble analytique de codimension pure $p$ de $D^{\prime}$; on dit que $V$ est à croisements normaux transverses à $\partial D$ si et seulement si, $\forall z^{0} \in V \cap \partial D$, il existe $f$ à croisements normaux transverses à $\partial D$ vérifiant $f^{-1}(0)=V$ dans un voisinage de $z^{0}$.

\section{Notations.}

- Pour $\alpha>0, \Lambda_{\alpha}(D)$ désigne l'intersection de l'ensemble des fonctions holomorphes sur $D$ avec l'espace de Lipschitz d'ordre $\alpha$ sur $D$.

- Pour $\alpha<0, B_{\alpha}(D)$ désigne l'espace des fonctions holomorphes sur $D$ vérifiant l'estimation suivante près du bord:

$$
\operatorname{Sup}_{z \in D}|f(z)| d_{z}^{-\alpha}<\infty
$$

où $d_{z}$ est la distance de $z$ au bord de $D$.

- On désigne par $B_{0}(D)$ l'espace des fonctions holomorphes sur $D$ vérifiant l'estimation:

$$
\operatorname{Sup}_{z \in D}|f(z)| \ln ^{-1}\left(d_{z}\right)<\infty
$$

- On note $A_{\alpha}(D)$, l'espace de fonctions suivant:

$$
\begin{cases}A_{\alpha}(D):=\Lambda_{\alpha}(D), & \text { si } \alpha>0 \\ A_{\alpha}(D):=B_{\alpha}(D), & \text { si } \alpha \leq 0\end{cases}
$$

- Pour $f$ une intersection complète, on note:

$$
\bar{\partial}\left[\frac{1}{f_{1}}\right] \wedge \cdots \wedge \bar{\partial}\left[\frac{1}{f_{p}}\right]:=\bar{\partial}\left[\frac{1}{f}\right]_{\{1, \ldots, p\}},
$$

le courant résiduel introduit par Coleff-Herrera dans [4].

- Pour $f$ une intersection complète sur $D$, on définit $n_{i}$ par

$$
n_{i}=\operatorname{Sup}_{z \in \partial D \cap f^{-1}(0)}\left(\operatorname{Ord}_{z} f_{i}\right), \quad \forall i \in\{1, \ldots, p\}
$$

où $\operatorname{Ord}_{z} f_{i}$ est l'ordre d'annulation de $f_{i}$ en $z$. 


\section{Principaux résultats}

Théorème 1.1. Soit $D$ un domaine borné de $\mathbb{C}^{n}$ strictement pseudoconvexe à bord $C^{\infty}$-lisse, soit $f=\left(f_{1}, \ldots, f_{p}\right)$ une intersection complète $\grave{a}$ croisements normaux transverses à $\partial D$. Alors si $g \in \Lambda_{\alpha}(D)$ et si le courant $g \bar{\partial}\left[\frac{1}{f}\right]_{\{1, \ldots, p\}}=0$ sur $D$, il existe des opérateurs linéaires bornés, $T_{i}$, de $\Lambda_{\alpha}(D)$ dans $A_{\alpha-\frac{n_{i}}{2}}(D)$ tels que $g=\sum_{i=1}^{n} f_{i} T_{i}(g)$.

Remarques. Pour $f$ une intersection complète sur $D, g \bar{\partial}\left[\frac{1}{f}\right]_{\{1, \ldots, p\}}=0$ si et seulement si $g$ est dans l'idéal engendré par $\left(f_{1}, \ldots, f_{p}\right)$; c'est un Théorème de Passare ([14]). Dans le cas d'une intersection complète à croisements normaux transverse à $\partial D$, il est assez facile de calculer $g \bar{\partial}\left[\frac{1}{f}\right]_{\{1, \ldots, p\}}$; on peut donc construire des champs de vecteurs sur $D$ et remplacer la condition $g \bar{\partial}\left[\frac{1}{f}\right]_{\{1, \ldots, p\}}=0$, par la condition $g$ est annulée par ces champs de vecteurs (voir $[\mathbf{7}]$ et $[\mathbf{8}]$ ).

Théorème 1.2. Sous les mêmes hypothèses qu'au Théorème 1.1; si g $\in$ $B_{\alpha}(D)(\alpha<0)$ et $g \bar{\partial}\left[\frac{1}{f}\right]_{\{1, \ldots, p\}}=0$, il existe des opérateurs linéaires bornés, $T_{i}^{\alpha}$, de $B_{\alpha}(D)$ dans $B_{\alpha-\frac{n_{i}}{2}}(D)$ tels que $g=\sum_{i=1}^{n} f_{i} T_{i}^{\alpha}(g)$.

Remarque. Nous retrouvons en corollaire du Théorème 1.1, des résultats de division dans l'algèbre $A^{\infty}(D):=C^{\infty}(\bar{D}) \cap \operatorname{Hol}(D)$.

Théorème 1.3. Soit $D$ un domaine borné de $\mathbb{C}^{n}$ strictement pseudoconvexe à bord $C^{\infty}$-lisse, soit $V$ un ensemble analytique à croisements normaux transverses à $\partial D$. Alors il existe un opérateur linéaire borné d'extension de $L^{\infty}(V \cap D) \cap \operatorname{Hol}(V \cap D)$ dans $L^{\infty}(D) \cap \operatorname{Hol}(D)$.

Remarques. Sans l'hypothèse $V$ à croisements normaux ce résultat est faux; en désignant par $B_{3}$ la boule de $\mathbb{C}^{3}$, on peut construire une fonction $f \in L^{\infty}\left(V \cap B_{3}\right) \cap \operatorname{Hol}\left(V \cap B_{3}\right)$ telle que $f$ n'a pas d'extension holomorphe dans $L^{2}\left(B_{3}\right) \cap \operatorname{Hol}\left(B_{3}\right)$ (voir $[\mathbf{6}]$ ). On peut démontrer également que le Théorème 1.3 reste vrai dans $L^{2}$ et remarquer que le Théorème de Berndtsson (voir [1]) ne permet pas d'obtenir ce résultat car les croisements normaux ne vérifient pas en général la condition d'intégrabilité de $[\mathbf{1}]$.

\section{Plan de la preuve des principaux résultats}

La preuve du Théorème 1.2 est parfaitement similaire à celle du Théorème 1.1, par conséquent, nous laissons le soin au lecteur d'adapter la démonstration du Théorème 1.1 à ce cas. Pour prouver les Théorèmes 1.1 et 1.3, nous allons raisonner localement en nous ramenant, par 
changement de coordonnées locales, à $f=\left(f_{1}, \ldots, f_{p}\right)$ avec $f_{i}$ qui est un monôme.

Définition 2.1. Soit $f=\left(f_{1}, \ldots, f_{p}\right)$ une intersection complète. On dit que $f$ est de type $(*)$ si et seulement si:

i) $\forall i \in\{1, \ldots, p\}, f_{i}(z)=z_{1}^{\alpha_{i, 1}} \times \cdots \times z_{n}^{\alpha_{i, n}}$, où $z_{j}$ sont les coordonnées standards de $\mathbb{C}^{n}$;

ii) $\forall I$, un sous-ensemble ordonné de $\{1, \ldots, p\}, f_{I}=\left(f_{i_{1}}, \ldots, f_{i_{k}}\right)$ est transverse à $\partial D$.

Pour localiser, nous allons utiliser un lemme de recouvrement initialement obtenu par A. Cumenge, mais adapté à notre situation dans [12].

Lemme 2.2. Pour tout $0<\varepsilon_{1}<\cdots<\varepsilon_{r_{0}}$, il existe $\left\{x_{i}\right\}_{i=1, \ldots, i_{0}}$ de $\partial D$ et des domaines $\left\{D_{i}^{r}\right\}_{i=1, \ldots, i_{0}}^{r=1, \ldots, r_{0}}$ avec $\Omega_{i}^{r}=D \cap D_{i}^{r}$ des domaines strictement pseudoconvexes à bord lisse tel que

i) $B\left(x_{i}, \varepsilon_{r-1}\right) \subset D_{i}^{r} \subset B\left(x_{i}, \varepsilon_{r}\right)$.

ii) $\partial D \subset \bigcup_{i=1}^{i_{0}} \Omega_{i}^{1}$.

iii) Si $i_{1}<i \leq i_{0}$, il existe $1 \leq j_{i} \leq p$ tel que $f_{j_{i}} \neq 0$ sur $D_{i}^{r}$ $\forall 1 \leq r \leq r_{0}$.

iv) Si $1 \leq i \leq i_{1}$ alors: $\Omega_{i}^{r} \cap f^{-1}(0) \neq \emptyset$ et sur $D_{i}^{r_{0}}$ il existe des coordonnées holomorphes telles que $f$ soit du type $(*)$ totalement transverse à $\partial \Omega_{i}^{r}, \forall 1 \leq r \leq r_{0}, \forall 1 \leq i \leq i_{1}$.

v) Si $1 \leq r \leq r^{\prime}$ et $\Omega_{i_{1}}^{r} \cap \cdots \cap \Omega_{i_{s}}^{r} \neq \emptyset$ alors il existe un domaine strictement pseudoconvexe $\Omega_{I=\left(i_{1}, \ldots, i_{s}\right)}^{r}$ à bord lisse tel que: $\Omega_{i_{1}}^{r} \cap \cdots \cap \Omega_{i_{s}}^{r} \subset \Omega_{I}^{r} \subset \Omega_{i_{1}}^{r^{\prime}} \cap \cdots \cap \Omega_{i_{s}}^{r^{\prime}}$; de plus si $\Omega_{I}^{r} \cap f^{-1}(0) \neq \emptyset$ alors $f^{-1}(0)$ est totalement transverse à $\partial \Omega_{I}^{r}$.

Le plan de l'article est le suivant: dans la partie 3 et 4, nous allons démontrer les Théorèmes 1.1 et 1.3 dans $\Omega_{I}^{r}$ avec $f$ de type $(*)$, ce qui est résumé par les propositions suivantes:

Proposition 2.3. Soit $D$ un domaine strictement pseudoconvexe à bord $C^{\infty}$-lisse, soit $f=\left(f_{1}, \ldots, f_{p}\right)$ une intersection complète du type $(*)$, alors $\forall g \in \Lambda_{\alpha}(D) \quad(\alpha>0)$ vérifiant $g \bar{\partial}\left[\frac{1}{f}\right]_{\{1, \ldots, p\}}=0$, il existe des opérateurs linéaires bornées, $T_{i}$, de $\Lambda_{\alpha}(D)$ dans $A_{\alpha-\frac{1}{2}} \sum_{j} \alpha_{i, j}(D)$ vérifiant les propriétés additionnelles: 
i) $z_{1}^{\beta_{i, 1}} \times \cdots \times z_{n}^{\beta_{i, n}} T_{i}(g)(z) \in A_{\alpha-\frac{1}{2}\left(\sum_{j} \alpha_{i, j}-\beta_{i, j}\right)}(D)$, si $\beta_{i, j} \leq \alpha_{i, j}$, $\forall j$.

ii) Soit $\theta=\left(\theta_{1}, \ldots, \theta_{n}\right)$ un n-uplet tel que $\forall \alpha=\left(\alpha_{1}, \ldots, \alpha_{n}\right)$ avec $\alpha_{j} \leq \theta_{j}, \forall j, z_{1}^{\alpha_{1}} \times \cdots \times z_{n}^{\alpha_{n}} g(z) \in \Lambda_{\alpha+\frac{1}{2} \sum_{j} \alpha_{j}}(D)$, alors $z_{1}^{\theta_{1}} \times \cdots \times z_{n}^{\theta_{n}} T_{i}(g) \in A_{\alpha-\frac{1}{2}\left(\sum_{j} \alpha_{i, j}-\theta_{j}\right)}(D)$.

Proposition 2.4. Soit $V$ un ensemble analytique de codimension pure $p$ sur un voisinage de $\bar{D}$. Supposons que $V:=\left\{z \in D^{\prime} / f_{1}(z)=\cdots=\right.$ $\left.f_{p}(z)=0\right\}$ avec $f=\left(f_{1}, \ldots, f_{p}\right)$ une intersection complète de type $(*)$. Alors, il existe un opérateur linéaire borné d'extension de $L^{\infty}(V \cap D) \cap$ $\operatorname{Hol}(V \cap D)$ dans $L^{\infty}(D) \cap \operatorname{Hol}(D)$.

Pour globaliser les solutions locales aux problèmes de division et d'extension, données par les Propositions 2.3 et 2.4, nous allons résoudre une succession de problèmes additifs de Cousin.

\section{Recollement des solutions du problème de division local.}

Soit $\left\{\Omega_{i}^{r_{0}}\right\}$ un recouvrement donné par le Lemme 2.2 avec $r_{0}$ fixé; sur $\Omega_{i}^{r_{0}}$, nous avons $f_{j_{i}} \neq \emptyset$ si $1 \leq i \leq i_{1}$ et $f$ totalement transverse du type $(*)$ si $i_{1}<i \leq i_{0}$, d'après la Proposition 2.3:

$$
g(z)=\sum_{l} f_{l} h_{i}^{l} \text { avec } h_{i}^{l} \in A_{\alpha-\alpha_{l}^{i}}\left(\Omega_{i}^{r_{0}}\right)
$$

où $\alpha_{l}^{i}=\frac{1}{2} \sum_{j} \alpha_{l, j}$ avec $f_{l}=z_{1}^{\alpha_{l, 1}} \times \cdots \times z_{n}^{\alpha_{l, n}}$ sur $\Omega_{i}^{r_{0}}$. Par conséquent $\operatorname{sur} \Omega_{i}^{r_{0}} \cap \Omega_{j}^{r_{0}}$ :

$$
0=\sum_{l} f_{l}\left(h_{i}^{1}-h_{j}^{l}\right)
$$

d'où $h_{i}^{1}-h_{j}^{1} \in I_{\left(f_{2}, \ldots, f_{p}\right)}\left(\Omega_{i}^{r_{0}} \cap \Omega_{j}^{r_{0}}\right)$. Soit $r<r_{0}$, d'après les propriétés du recouvrement, il existe $\Omega_{i j}^{r}$ avec $\Omega_{i}^{r} \cap \Omega_{j}^{r} \subset \Omega_{i j}^{r} \subset \Omega_{i}^{r_{0}} \cap \Omega_{j}^{r_{0}}$, appliquons de nouveau la Proposition 2.3, nous obtenons:

$$
h_{i}^{1}-h_{j}^{1}=\sum_{l \neq 1} f_{l} \lambda_{i j}^{r l},
$$

avec $\lambda_{i j}^{r l} \in A_{\alpha-\alpha_{1}^{i}-\alpha_{l}^{i}}\left(\Omega_{i j}^{r}\right)$ et de plus, $\lambda_{i j}^{r l}$ est un cocycle sur $\Omega_{i}^{r} \cap \Omega_{j}^{r}$. Prenons $\left(\chi_{\nu}\right)$ une partition de l'unité relative aux $D_{i}^{r}$ et écrivons $\lambda_{i}^{r l}=$ $\sum_{\nu} \chi_{\nu} \lambda_{\nu i}^{r l}+u^{l}$, où $u^{l}$ est une solution sur $D \operatorname{de} \bar{\partial} u^{l}=\sum_{\nu} \bar{\partial}\left(\chi_{\nu}\right) \lambda_{\nu i}^{r l}=\phi^{l}$; 
enfin considérons $G$ holomorphe sur $D$ définie par:

$$
\begin{aligned}
G & :=g-f_{1}\left(h_{i}^{1}+\sum_{l \neq 1} f_{l} \lambda_{i}^{r l}\right) \\
& :=\sum_{l \neq 1} f_{l}\left(h_{i}^{l}-f_{1} \lambda_{i}^{r l}\right) \\
& :=\sum_{l \neq 1} f_{l} H_{i}^{l} \operatorname{sur} \Omega_{i}^{r} .
\end{aligned}
$$

Pour conclure il faut choisir correctement $u^{l}$, la solution de $\bar{\partial} u^{l}=\phi^{l}$, ce qui est donné par la proposition:

Proposition 2.5. Soit $D$ un domaine strictement pseudoconvexe à bord $C^{\infty}$-lisse et $\left\{\Omega_{\nu}\right\}_{1}^{k}, \lambda_{\nu i} \in \operatorname{Hol}\left(\Omega_{\nu} \cap \Omega_{i}\right)$, les données d'un premier problème de Cousin sur $D$. Soit $\phi$ la $(0,1)$-forme régulière sur $D$ valant $\sum_{\nu} \bar{\partial}\left(\chi_{\nu}\right) \lambda_{\nu i}$ sur $\Omega_{i}$. Si $\phi \in A_{\alpha}(D)$ et s'il existe une boule de centre $z_{0} \in$ $\partial D$ et de rayon $r$ avec $B\left(z_{0}, r\right) \subset \Omega_{i}$ telle que: pour un $n$-uplet $\theta=$ $\left(\theta_{1}, \ldots, \theta_{n}\right), z_{1}^{\alpha_{1}} \times \cdots \times z_{n}^{\alpha_{n}} \lambda_{\nu i} \in A_{\alpha+\frac{1}{2} \sum_{j} \alpha_{j}}\left(B\left(z_{0}, r\right) \cap \Omega_{\nu}\right) \forall \alpha_{j} \leq \theta_{j}$ et $\forall \nu$, alors il existe une solution de l'équation $\bar{\partial} u=\phi$ dans $A_{\alpha+\frac{1}{2}}(D)$ avec $z_{1}^{\theta_{1}} \times \cdots \times z_{n}^{\theta_{n}} u(z) \in A_{\alpha+\frac{1}{2}\left(\sum_{j} \theta_{j}+1\right)}\left(B\left(z_{0}, r^{\prime}\right)\right), \forall r^{\prime}<r$.

Ce résultat sur le $\bar{\partial}$, s'obtient de manière similaire que le Lemme 4.4 de [12] (voir également le Lemme 2.2 et 2.5 de [12]). D'après les Propositions 2.3 et 2.5 , on peut choisir $u^{l}$ de sorte que:

i) $h_{i}^{1}+\sum_{l \neq 1} f_{l} \lambda_{i}^{r l} \in A_{\alpha-\alpha_{1}^{i}}\left(\Omega_{i}^{r^{\prime}}\right)$ avec $r^{\prime}<r$.

ii) $z_{1}^{\beta_{l, 1}} \times \cdots \times z_{n}^{\beta_{l, n}} H_{i}^{l} \in A_{\alpha-\alpha_{l}^{i}+\frac{1}{2} \sum_{j} \beta_{l, j}}\left(\Omega_{i}^{r^{\prime}}\right) \forall \beta_{l, j} \leq \alpha_{l, j}$, avec $r^{\prime}<r$.

En utilisant ces deux conditions, on peut itérer $(n-1)$ fois ce procédé et obtenir:

$$
g(z)=\sum_{l} f_{l} h_{l} \text { avec } h_{l} \in A_{\alpha-\alpha_{l}^{i}}\left(\Omega_{i}^{r}\right) \text { si } r \text { assez petit. }
$$




\section{Recollement des solutions du problème d'extension local.}

Considérons le recouvrement $\left\{\Omega_{i}^{r_{0}}\right\}$; posons si $i_{1}<i \leq i_{0}, g_{i}=0$ sur $\Omega_{i}^{r_{0}}$ et si $1 \leq i \leq i_{1}, g_{i}=f_{i}$ une extension bornée de $g$ à $\Omega_{i}^{r_{0}}$. Nous avons sur $\Omega_{i}^{r_{0}} \cap \Omega_{i}^{r_{0}}$,

$$
g_{i}-g_{j}=\sum_{l} f_{l} \lambda_{i j}^{r l} \text { avec } \lambda_{i j}^{r l} \in A_{-\alpha_{l}^{i}}\left(\Omega_{i j}^{r}\right)
$$

où $r<r_{0}$, et $\lambda_{i j}^{r l}$ est un cocycle sur $\Omega_{i}^{r} \cap \Omega_{j}^{r}$. Prenons $\left(\chi_{\nu}\right)$ une partition de l'unité relative aux $D_{i}^{r}$ et écrivons:

$$
\lambda_{i}^{r l}=\sum_{\nu} \chi_{\nu} \lambda_{\nu i}^{r l}+u^{l},
$$

où $u^{l}$ est une solution sur $D$ de $\bar{\partial} u^{l}=\sum_{\nu} \bar{\partial}\left(\chi_{\nu}\right) \lambda_{\nu i}^{r l}$; enfin considérons:

$$
\begin{aligned}
G & =g_{i}+\sum_{l} f_{l} \lambda_{i}^{r l} \operatorname{sur} \Omega_{i}^{r^{\prime}} \text { avec } r^{\prime}<r \\
& :=g_{i}+\sum_{l} f_{l}\left(\sum_{\nu} \chi_{\nu} \lambda_{\nu i}^{r l}\right)+\sum_{l} u^{l} f_{l} \\
& :=g_{i}+\sum_{\nu} \chi_{\nu} \sum_{l} f_{l} \lambda_{\nu i}^{r l}+\sum_{l} u^{l} f_{l} \\
& :=g_{i}+\sum_{\nu} \chi_{\nu}\left(g_{\nu}-g_{i}\right)+\sum_{l} u^{l} f_{l} .
\end{aligned}
$$

En choisissant $u^{l}$ donnée par la Proposition 2.5, nous obtenons $\sum f_{l} u^{l} \in$ $L^{\infty}\left(\Omega_{i}^{r}\right)$.

\section{Preuve de la Proposition 2.3}

Nous allons rappeler rapidement la construction de Passare dans [13] et $[\mathbf{1 4}]$.

Définition 3.1. Soit $\varepsilon_{i}: \mathbb{R} \rightarrow \mathbb{R}^{+}, \delta \mapsto \varepsilon_{i}(\delta), \forall i \in\{1, \ldots, p\}$ une application $\mathcal{C}^{\infty}$; on dit que $\varepsilon(\delta)=\left(\varepsilon_{1}(\delta), \ldots, \varepsilon_{p}(\delta)\right)$ est un pavé admissible si et seulement si:

$$
\left\{\begin{array}{l}
\lim _{\delta \rightarrow 0} \varepsilon_{i}(\delta)=0 \\
\lim _{\delta \rightarrow 0} \frac{\varepsilon_{i}(\delta)}{\left(\varepsilon_{i+1}(\delta)\right)^{q}}=0, \forall q \in \mathbb{N}, \forall i \in\{1, \ldots, p-1\} .
\end{array}\right.
$$


Considérons, $\Xi: \mathbb{R}^{+*} \rightarrow \mathbb{R}$ une fonction $\mathcal{C}^{\infty}$-croissante, vérifiant

$$
\left\{\begin{array}{l}
\Xi(x)=1, \quad \text { si } x \geq 1 \\
\Xi(x)=0, \quad \text { si } x \leq \frac{1}{2}
\end{array}\right.
$$

Pour $\left(f_{1}, \ldots, f_{p}\right), p$ fonctions holomorphes sur un voisinage de $\bar{D}$ et $I=\left\{i_{1}, \ldots, i_{q}\right\}, J=\left\{j_{1}, \ldots, j_{r}\right\}$ deux sous-ensembles disjoints de $\{1, \ldots, p\}$, on considère la forme différentielle:

$\left[\frac{\Xi^{\varepsilon}}{f}\right]_{I} \bar{\partial}\left[\frac{\Xi^{\varepsilon}}{f}\right]_{J}:=\frac{\Xi\left(\frac{\left|f_{i_{1}}\right|}{\varepsilon_{i_{1}}}\right) \times \cdots \times \Xi\left(\frac{\left|f_{i_{q}}\right|}{\varepsilon_{i_{q}}}\right) \times \bar{\partial}\left(\Xi\left(\frac{\left|f_{j_{1}}\right|}{\varepsilon_{j_{1}}}\right)\right) \wedge \cdots \wedge \bar{\partial}\left(\Xi\left(\frac{\left|f_{j_{r}}\right|}{\varepsilon_{j_{r}}}\right)\right)}{f_{i_{1}} \times \cdots \times f_{i_{q}} \times f_{j_{1}} \times \cdots \times f_{j_{r}}}$.

Théorème 3.2 (Passare $[\mathbf{1 3}]) . \forall I, J,\left[\frac{\Xi^{\varepsilon}}{f}\right]_{I} \bar{\partial}\left[\frac{\Xi^{\varepsilon}}{f}\right]_{J}$ converge faiblement sur $D$ quand $\delta \mapsto 0$. Si l'on note $\left[\frac{1}{f}\right]_{I} \bar{\partial}\left[\frac{1}{f}\right]_{J}$ le courant limite, alors $\left[\frac{1}{f}\right]_{I} \bar{\partial}\left[\frac{1}{f}\right]_{J}$ est un courant d'ordre fini.

Remarque. En général, le courant $\left[\frac{1}{f}\right]_{I} \bar{\partial}\left[\frac{1}{f}\right]_{J}$ dépend de $\varepsilon$, le pavé admissible et de $\Xi$. Mais, dans le cas où $f$ est à croisements normaux (voir par exemple, $[\mathbf{7}]$ et $[\mathbf{8}]$ ) $\left[\frac{1}{f}\right]_{I} \bar{\partial}\left[\frac{1}{f}\right]_{J}$ sont des courants canoniques (indépendants de $\varepsilon$ et $\Xi$ ) qui conïncident avec les courants définis par Coleff-Herrera dans [4].

Théorème 3.3 (Passare $[\mathbf{1 3}]$ ). Soit $D$ un domaine strictement pseudoconvexe de $\mathbb{C}^{n}$ à bord $\mathcal{C}^{\infty}$-lisse, soit $f=\left(f_{1}, \ldots, f_{p}\right)$ vérifiant $\left\{f_{i_{1}}, \ldots, f_{i_{k}}\right\}$ est une intersection complète sur un voisinage de $\bar{D}$ pour tout $\left\{i_{1}, \ldots, i_{k}\right\}$ un multi-indice à valeurs dans $\{1, \ldots, p\}$ et $g \in \mathcal{C}^{\infty}(\bar{D})$, alors, pour tout $z \in D$ :

$$
\begin{array}{r}
g(z)=\sum_{k=0}^{p-1} \sum_{|I|=p-k,|J|=k} C_{n, k}[f(z)]_{I}\left\langle g(\zeta)\left[\frac{1}{f}\right]_{I} \bar{\partial}\left[\frac{1}{f}\right]_{J},\right. \\
\left.(b(\zeta, z))_{J} \wedge A^{N, n-k}(\zeta, z)\right\rangle \\
+C_{n}\left\langle g(\zeta) \bar{\partial}\left[\frac{1}{f}\right]_{\{1, \ldots, p\}},\right. \\
\left.(b(\zeta, z))_{\{1, \ldots, p\}} \wedge A^{N, n-p}(\zeta, z)\right\rangle,
\end{array}
$$


où $[f(z)]_{I}=f_{i_{1}}(z) \times \cdots \times f_{i_{p-k}}(z),(b(\zeta, z))_{J}=b_{j_{1}}(\zeta, z) \wedge \cdots \wedge b_{j_{k}}(\zeta, z)$ avec $b_{j}(\zeta, z)=\sum_{l=1}^{n} B_{j l}(\zeta, z) d \zeta_{l}$ et $f_{j}(z)-f_{j}(\zeta)=\sum_{l} B_{j l}(\zeta, z)\left(z_{l}-\zeta_{l}\right)$ sur $D^{\prime}$, enfin

$$
A^{N, n-k}(\zeta, z)=\left(\frac{\rho(\zeta)}{\rho(\zeta)+\langle h(\zeta, z), \zeta-z\rangle}\right)^{N+n-k}(\bar{\partial}(\tilde{h}(\zeta, z)))^{n-k}
$$

où

- $h_{j}: \bar{D} \times \bar{D} \rightarrow \mathbb{C}$, les fonctions supports définies par Fornaess dans $[\mathbf{1 0}]$.

- $h(\zeta, z)=\left(h_{1}(\zeta, z), \ldots, h_{n}(\zeta, z)\right)$.

- $\tilde{h}(\zeta, z)=\frac{1}{\rho(\zeta)} \sum_{i=1}^{n} h_{i}(\zeta, z) d \zeta_{i}$.

- $N$ est un réel quelconque supérieur à l'ordre des courants $\left[\frac{1}{f}\right]_{I} \bar{\partial}\left[\frac{1}{f}\right]_{J}$, $\forall I, J$.

Corollaire 3.4 (Passare $[14]$ ).

$$
g \in I_{\left(f_{1}, \ldots, f_{p}\right)}(D) \text { si et seulement si } g \bar{\partial}\left[\frac{1}{f}\right]_{\{1, \ldots, p\}}=0 \text {. }
$$

Dans le cas d'une intersection complète à croisements normaux, nous pouvons calculer assez facilement les courants $\left[\frac{1}{f}\right]_{I} \bar{\partial}\left[\frac{1}{f}\right]_{J}, \forall I, J$. Pour une intersection à croisements normaux de codimension 1 , Dolbeault dans $[\mathbf{7}]$ et $[\mathbf{9}]$ explicite $\left[\frac{1}{f}\right]$ et $\bar{\partial}\left[\frac{1}{f}\right]$; nous allons généraliser pour la codimension supérieure à 1 .

Proposition 3.5. Soit $\varphi$ une fonction $\mathcal{C}^{\infty}$ à support compact dans $\mathbb{C}$, $\alpha \in \mathbb{N}^{*}$ alors nous avons:

i) $\lim _{\varepsilon \rightarrow 0} \int_{|z|=\varepsilon} \frac{\varphi}{z^{\alpha}} d z=2 i \pi \frac{\partial^{\alpha-1} \varphi}{\partial z^{\alpha-1}}(0)$.

ii) $\lim _{\varepsilon \rightarrow 0} \int_{|z| \geq \varepsilon} \frac{\varphi}{z^{\alpha}} d z \wedge d \bar{z}=\frac{1}{(\alpha-1) !} \int_{\mathbb{C}} \frac{1}{z} \frac{\partial^{\alpha-1} \varphi}{\partial z^{\alpha-1}}(z) d z \wedge d \bar{z}$.

On peut trouver ces deux égalités très classiques, par exemple dans Passare [14] et Dolbeault [7] et [9].

Nous allons commencer par démontrer le résultat principal de la Proposition 2.3 à savoir: l'existence des opéateurs linéaires de $\Lambda_{\alpha}(D)$ dans $A_{\alpha-\frac{1}{2}} \sum_{j} \alpha_{i, j}(D)$, solutions du problème de division dans la cas $f$ de type $(*)$ (nous indiquerons à la fin comment vérifier les propriétés i) et ii)). Pour éviter les notations superflues nuisant à la compréhension des arguments, nous allons considérer le cas $f=\left(f_{1}, f_{2}\right)$ avec $f_{1}=z_{1}^{\alpha_{1}} z_{2}^{\alpha_{2}}$, 
$f_{2}=z_{3}^{\beta_{1}} z_{4}^{\beta_{2}}$, clairement le cas général s'obtient de la même manière. On remarque que l'hypothèse de transversalité entraîne que $n>4$; soit $g \in \Lambda_{\alpha}(D)(\alpha>0)$ telle que $g \bar{\partial}\left[\frac{1}{f}\right]_{\{1,2\}}=0$, par un procédé standard de régularisation, nous pouvons lui appliquer le Théorème 3.3 avec $N>\sum \alpha_{i}+\beta_{i}$, nous obtenons:

$$
\begin{aligned}
g(z)= & f_{1}(z) f_{2}(z)\left\langle g(\zeta)\left[\frac{1}{f}\right]_{\{1,2\}}, A^{N, n}(\zeta, z)\right\rangle \\
& +f_{1}(z)\left\langle g(\zeta)\left[\frac{1}{f}\right]_{\{1\}} \bar{\partial}\left[\frac{1}{f}\right]_{\{2\}},(b(\zeta, z))_{2} A^{N, n-1}(\zeta, z)\right\rangle \\
& +f_{2}(z)\left\langle g(\zeta)\left[\frac{1}{f}\right]_{\{2\}} \bar{\partial}\left[\frac{1}{f}\right]_{\{1\}},(b(\zeta, z))_{1} A^{N, n-1}(\zeta, z)\right\rangle \\
= & f_{1}(z) f_{2}(z) \lim _{\delta \rightarrow 0} \int_{D \cap\left\{\left|f_{1}\right| \geq \varepsilon_{1}(\delta),\left|f_{2}\right| \geq \varepsilon_{2}(\delta)\right\}} \frac{g(\zeta)}{f_{1}(\zeta) f_{2}(\zeta)} A^{N, n}(\zeta, z) \\
& +f_{1}(z) \lim _{\delta \rightarrow 0} \int_{D \cap\left\{\left|f_{1}\right| \geq \varepsilon_{1}(\delta),\left|f_{2}\right|=\varepsilon_{2}(\delta)\right\}} \frac{g(\zeta)}{f_{1}(\zeta) f_{2}(\zeta)}(b(\zeta, z))_{2} A^{N, n-1}(\zeta, z) \\
& +f_{2}(z) \lim _{\delta \rightarrow 0} \int_{D \cap\left\{\left|f_{1}\right|=\varepsilon_{1}(\delta),\left|f_{2}\right| \geq \varepsilon_{2}(\delta)\right\}} \frac{g(\zeta)}{f_{1}(\zeta) f_{2}(\zeta)}(b(\zeta, z))_{1} A^{N, n-1}(\zeta, z)
\end{aligned}
$$

(car dans ce cas, les courants de Passare coïncident avec ceux de ColeffHerrera de [4]). De plus, $f$ étant une intersection complète du type (*), les limites existent sans aucune restriction, soit:

$$
\begin{aligned}
g(z)= & f_{1}(z) f_{2}(z) \lim _{\left(\varepsilon_{1}, \varepsilon_{2}\right) \rightarrow 0} \int_{D \cap\left\{\left|f_{1}\right| \geq \varepsilon_{1},\left|f_{2}\right| \geq \varepsilon_{2}\right\}} \frac{g(\zeta)}{f_{1}(\zeta) f_{2}(\zeta)} A^{N, n}(\zeta, z) \\
& +f_{1}(z) \lim _{\left(\varepsilon_{1}, \varepsilon_{2}\right) \rightarrow 0} \int_{D \cap\left\{\left|f_{1}\right| \geq \varepsilon_{1},\left|f_{2}\right|=\varepsilon_{2}\right\}} \frac{g(\zeta)}{f_{1}(\zeta) f_{2}(\zeta)}(b(\zeta, z))_{\{2\}} A^{N, n-1}(\zeta, z) \\
& +f_{2}(z) \lim _{\left(\varepsilon_{1}, \varepsilon_{2}\right) \rightarrow 0} \int_{D \cap\left\{\left|f_{1}\right|=\varepsilon_{1},\left|f_{2}\right| \geq \varepsilon_{2}\right\}} \frac{g(\zeta)}{f_{1}(\zeta) f_{2}(\zeta)}(b(\zeta, z))_{\{1\}} A^{N, n-1}(\zeta, z),
\end{aligned}
$$


il n'est pas difficile de voir (par exemple dans [8]), en posant

$D_{I=\left(i_{1}, \ldots, i_{q}\right)}^{J=\left(j_{1}, \ldots, j_{r}\right)}:=D \cap\left\{\left|z_{i_{1}}\right| \geq \varepsilon_{i_{1}}, \ldots,\left|z_{i_{q}}\right| \geq \varepsilon_{i_{q}},\left|z_{j_{1}}\right|=\varepsilon_{j_{1}}, \ldots,\left|z_{j_{r}}\right|=\varepsilon_{j_{r}}\right\}$

It. $f\left(\varepsilon_{1}, \varepsilon_{2}, \varepsilon_{3}, \varepsilon_{4}\right)=\lim _{\varepsilon_{1} \rightarrow 0}\left[\lim _{\varepsilon_{2} \rightarrow 0}\left[\lim _{\varepsilon_{3} \rightarrow 0}\left[\lim _{\varepsilon_{4} \rightarrow 0} f\left(\varepsilon_{1}, \varepsilon_{2}, \varepsilon_{3}, \varepsilon_{4}\right)\right]\right]\right]$,

(l'ordre dans lequel nous prendons les limites par la suite n'a pas d'importance car les résidus qui nous concernent ont une structure très simple) que nous avons:

$$
\begin{aligned}
& \lim _{\left(\varepsilon_{1}, \varepsilon_{2}\right) \rightarrow 0} \int_{D \cap\left\{\left|f_{1}\right| \geq \varepsilon_{1},\left|f_{2}\right| \geq \varepsilon_{2}\right\}} \frac{g(\zeta)}{f_{1}(\zeta) f_{2}(\zeta)} A^{N, n}(\zeta, z) \\
&= \text { It. } \int_{D_{(1,2,3,4)}^{\emptyset}} \frac{g(\zeta)}{\zeta_{1}^{\alpha_{1}} \zeta_{2}^{\alpha_{2}} \zeta_{3}^{\beta_{1}} \zeta_{4}^{\beta_{2}}} A^{N, n}(\zeta, z) \\
& \lim _{\left(\varepsilon_{1}, \varepsilon_{2}\right) \rightarrow 0} \int_{D \cap\left\{\left|f_{1}\right| \geq \varepsilon_{1},\left|f_{2}\right|=\varepsilon_{2}\right\}} \frac{g(\zeta)}{f_{1}(\zeta) f_{2}(\zeta)}(b(\zeta, z))_{\{2\}} A^{N, n-1}(\zeta, z) \\
&=\text { It. } \int_{D_{(1,2,3)}^{(4)}} \frac{g(\zeta)}{\zeta_{1}^{\alpha_{1}} \zeta_{2}^{\alpha_{2}} \zeta_{3}^{\beta_{1}} \zeta_{4}^{\beta_{2}}}(b(\zeta, z))_{\{2\}} A^{N, n-1}(\zeta, z) \\
& \quad+\text { It. } \int_{D_{(1,2,4)}^{(3)}} \frac{g(\zeta)}{\zeta_{1}^{\alpha_{1}} \zeta_{2}^{\alpha_{2}} \zeta_{3}^{\beta_{1}} \zeta_{4}^{\beta_{2}}}(b(\zeta, z))_{\{2\}} A^{N, n-1}(\zeta, z)
\end{aligned}
$$

et

$$
\begin{aligned}
\lim _{\left(\varepsilon_{1}, \varepsilon_{2}\right) \rightarrow 0} & \int_{D \cap\left\{\left|f_{1}\right|=\varepsilon_{1},\left|f_{2}\right| \geq \varepsilon_{2}\right\}} \frac{g(\zeta)}{f_{1}(\zeta) f_{2}(\zeta)}(b(\zeta, z))_{\{1\}} A^{N, n-1}(\zeta, z) \\
= & \text { It. } \int_{D_{(2,3,4)}^{(1)}} \frac{g(\zeta)}{\zeta_{1}^{\alpha_{1}} \zeta_{2}^{\alpha_{2}} \zeta_{3}^{\beta_{1}} \zeta_{4}^{\beta_{2}}}(b(\zeta, z))_{\{1\}} A^{N, n-1}(\zeta, z) \\
& + \text { It. } \int_{D_{(1,3,4)}^{(2)}} \frac{g(\zeta)}{\zeta_{1}^{\alpha_{1}} \zeta_{2}^{\alpha_{2}} \zeta_{3}^{\beta_{1}} \zeta_{4}^{\beta_{2}}}(b(\zeta, z))_{\{1\}} A^{N, n-1}(\zeta, z)
\end{aligned}
$$




\section{Estimation des termes.}

$$
\begin{aligned}
& z_{1}^{\alpha_{1}} z_{2}^{\alpha_{2}} \text { It. } \int_{D_{(1,2,3,4)}^{\emptyset}} \frac{g(\zeta)}{\zeta_{1}^{\alpha_{1}} \zeta_{2}^{\alpha_{2}} \zeta_{3}^{\beta_{1}} \zeta_{4}^{\beta_{2}}} A^{N, n}(\zeta, z), \\
& \text { It. } \int_{D_{(1,2,3)}^{4}} \frac{g(\zeta)}{\zeta_{1}^{\alpha_{1}} \zeta_{2}^{\alpha_{2}} \zeta_{3}^{\beta_{1}} \zeta_{4}^{\beta_{2}}}(b(\zeta, z))_{\{2\}} A^{N, n-1}(\zeta, z) .
\end{aligned}
$$

Par des arguments standards (voir par exemple [3]), pour estimer les termes ci-dessus, il suffit d'avoir des estimations sur les deux termes suivants:

$$
\begin{aligned}
& z_{1}^{\alpha_{1}} z_{2}^{\alpha_{2}} \text { It. } \int_{D_{(1,2,3,4)}^{\emptyset} \cap B(q, r)} \frac{g(\zeta)}{\zeta_{1}^{\alpha_{1}} \zeta_{2}^{\alpha_{2}} \zeta_{3}^{\beta_{1}} \zeta_{4}^{\beta_{2}}} A^{N, n}(\zeta, z), \\
& \text { It. } \int_{D_{(1,2,3)}^{4} \cap B(q, r)} \frac{g(\zeta)}{\zeta_{1}^{\alpha_{1}} \zeta_{2}^{\alpha_{2}} \zeta_{3}^{\beta_{1}} \zeta_{4}^{\beta_{2}}}(b(\zeta, z))_{\{2\}} A^{N, n-1}(\zeta, z),
\end{aligned}
$$

quand $z \in B\left(q, \frac{r}{2}\right)$, où $B(q, r)$ est une boule centrée en $q \in \partial D$ de rayon $r$ qui sera choisi ultérieurement. Avec l'hypothèse de totale transversalité, on peut se restreindre à $q \in \partial D \cap\left\{\zeta_{1}=\zeta_{2}=\zeta_{3}=\zeta_{4}=0\right\}$ (si $q \notin \partial D \cap\left\{\zeta_{1}=\zeta_{2}=\zeta_{3}=\zeta_{4}=0\right\}$, l'estimation est meilleure). L'hypothèse de transversalité entraîne qu'il existe $\zeta_{j}$ avec $j \notin\{1, \ldots, 4\}$ (on rappelle que l'hypothèse de transversalité implique $n>4$ ) tel que $\frac{\partial \rho}{\partial \zeta_{j}}(q) \neq 0$; on va supposer que $j=n$ et prenons $r$ tel que $\left|\frac{\partial \rho}{\partial \zeta_{n}}(\zeta)\right| \geq C$ sur $B(q, r)$.

$$
\begin{aligned}
& D^{\Gamma} f_{1}=\sum_{\Gamma_{1}+\Gamma_{2}=\Gamma} D^{\Gamma_{1}}\left(z_{1}^{\alpha_{1}} z_{2}^{\alpha_{2}}\right) \int_{D_{(1,2,3,4}^{\emptyset} \cap B(q, r)} \frac{g(\zeta)}{\zeta_{1}^{\alpha_{1}} \zeta_{2}^{\alpha_{2}} \zeta_{3}^{\beta_{1}} \zeta_{4}^{\beta_{2}}} D^{\Gamma_{2}}\left(A^{N, n}(\zeta, z)\right)\left(I_{1}\right) \\
& D^{\Gamma_{2}} f_{2}=\int_{D_{(1,2,3)}^{4} \cap B(q, r)} \frac{g(\zeta)}{\zeta_{1}^{\alpha_{1}} \zeta_{2}^{\alpha_{2}} \zeta_{3}^{\beta_{1}} \zeta_{4}^{\beta_{2}}} D^{\Gamma}(b(\zeta, z))_{\{2\}}\left(A^{N, n-1}(\zeta, z)\right)\left(I_{2}\right) .
\end{aligned}
$$

Par soucis de clarité, dans $\left(I_{1}\right)$, nous allons estimer les termes de la somme avec $\Gamma_{1}=0$; si $\Gamma_{1} \neq 0$, la démonstration est similaire; de plus, nous notons

$$
\left\{\begin{array}{l}
D^{\emptyset}:=D_{(1,2,3,4)}^{\emptyset} \cap B(q, r) \\
D^{(4)}:=D_{(1,2,3)}^{(4)} \cap B(q, r) .
\end{array}\right.
$$

Par des intégrations par parties successives (voir [3]), nous avons que:

$$
\int_{D_{(1,2,3,4)}^{\emptyset} \cap B(q, r)} \frac{g(\zeta)}{\zeta_{1}^{\alpha_{1}} \zeta_{2}^{\alpha_{2}} \zeta_{3}^{\beta_{1}} \zeta_{4}^{\beta_{2}}} D^{\Gamma}\left(A^{N, n}(\zeta, z)\right)
$$


est majorée par le module d'intégrale du type:

$$
\begin{aligned}
\int_{D^{\emptyset}} \frac{\partial^{\lambda} g}{\partial \zeta_{n}^{\lambda}}(\zeta) & \frac{1}{\zeta_{1}^{\alpha_{1}} \zeta_{2}^{\alpha_{2}} \zeta_{3}^{\beta_{1}} \zeta_{4}^{\beta_{2}}} \\
& \times \frac{(\rho(\zeta))^{N-1+\lambda}}{(\rho(\zeta)+\langle h(\zeta, z), \zeta-z\rangle)^{N+n+|\Gamma|}} G_{1}^{\lambda}(\zeta, z), \forall \lambda \geq 0,\left(I_{3}\right)
\end{aligned}
$$

où $G_{1}^{\lambda}(\zeta, z)$ est une forme de classe $\mathcal{C}^{\infty}(\bar{D} \times \bar{D})$; de même

$$
\int_{D^{(4)}} \frac{g(\zeta)}{\zeta_{1}^{\alpha_{1}} \zeta_{2}^{\alpha_{2}} \zeta_{3}^{\beta_{1}} \zeta_{4}^{\beta_{2}}} D^{\Gamma}\left((b(\zeta, z))_{\{2\}} A^{N, n-1}(\zeta, z)\right)
$$

est majorée par le module d'intégrale du type:

$$
\begin{aligned}
\int_{D^{(4)}} \frac{\partial^{\lambda} g}{\partial \zeta_{n}^{\lambda}}(\zeta) \frac{1}{\zeta_{1}^{\alpha_{1}} \zeta_{2}^{\alpha_{2}} \zeta_{3}^{\beta_{1}} \zeta_{4}^{\beta_{2}}} \\
\quad \times \frac{(\rho(\zeta))^{N-1+\lambda}(b(\zeta, z))_{\{2\}}}{(\rho(\zeta)+\langle h(\zeta, z), \zeta-z\rangle)^{N+n-1+|\Gamma|}} G_{2}^{\lambda}(\zeta, z), \forall \lambda \geq 0,\left(I_{4}\right)
\end{aligned}
$$

où $G_{2}^{\lambda}(\zeta, z)$ est une forme de classe $\mathcal{C}^{\infty}(\bar{D} \times \bar{D})$. On va supposer que

$$
\left\{\begin{array}{l}
\alpha>\frac{n_{1}}{2}=\frac{\alpha_{1}+\alpha_{2}}{2} \\
\alpha>\frac{n_{2}}{2}=\frac{\beta_{1}+\beta_{2}}{2}
\end{array}\right.
$$

et on va montrer que: $\int_{D^{\emptyset}}$ et $\int_{D^{(4)}}$ sont dans $\Lambda_{\alpha-\frac{n_{i}}{2}}(D)$ (les autres cas se traitent de manière similaire). Il faut estimer $z_{1}^{\alpha_{1}} z_{2}^{\alpha_{2}}\left(I_{3}\right)$ et $\left(I_{4}\right)$; à ces fins, remarquons que

$$
z_{1}^{\alpha_{1}} z_{2}^{\alpha_{2}}=\sum_{j, l} \zeta_{1}^{\alpha_{1}-j} \zeta_{2}^{\alpha_{2}-l} \mathbf{O}\left(|\zeta-z|^{j+l}\right)
$$

et si l'on construit $(b(\zeta, z))_{\{2\}}$ avec la formule de Taylor alors $(b(\zeta, z))_{\{2\}}=b_{1}(\zeta, z) d \zeta_{3}+b_{2}(\zeta, z) d \zeta_{4}$ avec

$$
\left\{\begin{array}{l}
b_{1}(\zeta, z)=\sum_{j, l} \zeta_{3}^{\beta_{1}-j} \zeta_{4}^{\beta_{2}-l} \mathbf{O}\left(|\zeta-z|^{j+l-1}\right) \\
b_{2}(\zeta, z)=\sum_{j, l} \zeta_{3}^{\beta_{1}-j} \zeta_{4}^{\beta_{2}-l} \mathbf{O}\left(|\zeta-z|^{j+l-1}\right)
\end{array}\right.
$$


Tenant compte de ceci $z_{1}^{\alpha_{1}} z_{2}^{\alpha_{2}}\left(I_{3}\right)$ et $\left(I_{4}\right)$ sont majorées par une somme d'intégrales du type:

$$
\begin{aligned}
\int_{D^{\emptyset}} & \frac{\partial^{\lambda} g}{\partial \zeta_{n}^{\lambda}}(\zeta) \frac{\mathbf{O}\left(|\zeta-z|^{j+l}\right)}{\zeta_{1}^{j} \zeta_{2}^{l} \zeta_{3}^{\beta_{1}} \zeta_{4}^{\beta_{2}}} \\
& \times \frac{(\rho(\zeta))^{N-1+\lambda}}{(\rho(\zeta)+\langle h(\zeta, z), \zeta-z\rangle)^{N+n+|\Gamma|}} G_{1}^{\lambda}(\zeta, z), \quad \forall \lambda \geq 0,\left(I_{5}\right) \\
\int_{D^{(4)}} & \frac{\partial^{\lambda} g}{\partial \zeta_{n}^{\lambda}}(\zeta) \frac{\mathbf{O}\left(|\zeta-z|^{j+l-1}\right)}{\zeta_{1}^{\alpha_{1}} \zeta_{2}^{\alpha_{2}} \zeta_{3}^{j} \zeta_{4}^{l}} \\
& \times \frac{(\rho(\zeta))^{N-1+\lambda}}{(\rho(\zeta)+\langle h(\zeta, z), \zeta-z\rangle)^{N+n-1+|\Gamma|}} G_{2}^{\lambda}(\zeta, z), \quad \forall \lambda \geq 0,\left(I_{6}\right) .
\end{aligned}
$$

Posons maintenant le changement de variable sur $\mathbb{C}^{n}$, pour $\zeta \in B(q, r)$ :

$$
\left\{\begin{array}{l}
\zeta_{1}^{\prime}=\zeta_{1} \\
\vdots \\
\zeta_{n-1}^{\prime}=\zeta_{n-1} \\
\zeta_{n}^{\prime}=\frac{\partial \rho}{\partial \zeta_{1}}(z) \zeta_{1}+\cdots+\frac{\partial \rho}{\partial \zeta_{n}}(z) \zeta_{n}
\end{array}\right.
$$

dans ces coordonnées, $\frac{\partial}{\partial \zeta_{i}^{\prime}}$ avec $i \in\{1, \ldots, n-1\}$, peut s'écrire $L_{i}^{\prime}+V_{i}^{\prime}$ où $L_{i}^{\prime}$ est un $(1,0)$ champ de vecteurs tangent à $\{\rho=$ cte. $\}$ et $V_{i}^{\prime}$ est un champ de vecteurs vérifiant $V_{i}^{\prime}(z)=0$. Par la Proposition 3.5, en revenant dans les coordonnées standards:

$$
\begin{aligned}
\left(I_{5}\right) \lesssim \mid \int_{D^{\emptyset}} \frac{1}{\zeta_{1} \zeta_{2} \zeta_{3} \zeta_{4}}\left[L_{1}+V_{1}\right]^{j-1} \ldots\left[L_{4}+V_{4}\right]^{\beta_{2}-1} \\
\\
\quad \times\left(\frac{\frac{\partial^{\lambda} g}{\partial \zeta_{n}^{\lambda}}(\zeta)(\rho(\zeta))^{N-1+\lambda} G_{1}^{\lambda}(\zeta, z) \mathbf{O}\left(|\zeta-z|^{j+l}\right)}{\left.\rho(\zeta)+\langle h(\zeta, z), \zeta-z\rangle)^{N+n+|\Gamma|}\right) \mid}\right.
\end{aligned}
$$


pour tout $\lambda \geq 0$,

$$
\begin{aligned}
\left(I_{6}\right) \lesssim \mid \int_{D^{(4)} \cap\left\{\zeta_{4}=0\right\}} & \frac{1}{\zeta_{1} \zeta_{2} \zeta_{3}}\left[L_{1}+V_{1}\right]^{\alpha_{1}-1} \ldots\left[L_{4}+V_{4}\right]^{l-1} \\
& \times\left(\frac{\frac{\partial^{\lambda} g}{\partial \zeta_{n}^{\lambda}}(\zeta)(\rho(\zeta))^{N-1+\lambda} G_{2}^{\lambda}(\zeta, z) \mathbf{O}\left(|\zeta-z|^{j+l-1}\right)}{(\rho(\zeta)+\langle h(\zeta, z), \zeta-z\rangle)^{N+n+|\Gamma|-1}}\right) \mid
\end{aligned}
$$

pour tout $\lambda \geq 0$, où $L_{i}=A L_{i}^{\prime}$ et $V_{i}=A V_{i}^{\prime} \forall i \in\{1,2,3,4\}$ ( $A$ est la matrice de passage des coordonnées $(\zeta)^{\prime}$ aux coordonnées $\left.(\zeta)\right)$. $L_{i}$ sont des $(1,0)$ champs de vecteurs tangents à $\{\rho=$ cte. $\}$ et $V_{i}(z)=0$.

Lemme 3.6. Soit $u$ une fonction holomorphe dans $D$ et $\alpha>0$, alors nous avons les équivalences suivantes:

$u \in \Lambda_{\alpha}(D)$

pour tout $k \in N^{*}, k>\alpha$, pour tout $z \in D,\left|\nabla^{k} u(z)\right| \leq C(-\rho(z))^{\alpha-k}$.

Il existe $k \in N^{*}, k>\alpha$, pour tout $z \in D,\left|\nabla^{k} u(z)\right| \leq C(-\rho(z))^{\alpha-k}$.

Ce qui précéde et le Lemme 3.6 entraînent:

- $L_{i}(\rho(\zeta)+\langle h(\zeta, z), \zeta-z\rangle)=\mathbf{O}(|\zeta-z|)$,

$\operatorname{car}\langle h(\zeta, z), \zeta-z\rangle=\langle\partial \rho(\zeta), \zeta-z\rangle+\mathbf{O}\left(|\zeta-z|^{2}\right)$,

- $L_{i}(h)=\mathbf{O}\left(|\rho(\zeta)|^{-a-\frac{1}{2}}\right)$ si $h \in \operatorname{Hol}(D)$ et $h=\mathbf{O}\left(|\rho(\zeta)|^{-a}\right)(a>0)$,

- $V_{i}(f)=\mathbf{O}(|\operatorname{grad}(f)||\zeta-z|)$,

- $\left(-\rho(\zeta)-\rho(z)+|\zeta-z|^{2}+|\operatorname{Im}\langle h(\zeta, z), \zeta-z\rangle|\right) \lesssim|\rho(\zeta)+\langle h(\zeta, z), \zeta-z\rangle|$,

- si $\lambda>\alpha$ alors $\frac{\partial^{\lambda} g}{\partial \zeta_{n}^{\lambda}}=\mathbf{O}\left(|\rho(\zeta)|^{\alpha-\lambda}\right)$ d'après le lemme d'HardyLittlewood,

d'où:

$$
\begin{aligned}
& \left(I_{5}\right) \lesssim \int_{D^{\emptyset}} \frac{d v}{\left(\begin{array}{c}
\left|\zeta_{1} \zeta_{2} \zeta_{3} \zeta_{4}\right|\left(-\rho(\zeta)-\rho(z)+|\zeta-z|^{2}\right. \\
+|\operatorname{Im}\langle h(\zeta, z), \zeta-z\rangle|)^{n+|\Gamma|-\alpha+\frac{\beta_{1}+\beta_{2}}{2}-1}
\end{array}\right)} \\
& \left(I_{6}\right) \lesssim \int_{D^{(4)} \cap\left\{\zeta_{4}=0\right\}} \frac{d v}{\left(\begin{array}{c}
\left|\zeta_{1} \zeta_{2} \zeta_{3}\right|\left(-\rho(\zeta)-\rho(z)+|\zeta-z|^{2}\right. \\
+|\operatorname{Im}\langle h(\zeta, z), \zeta-z\rangle|)^{n+|\Gamma|-\alpha+\frac{\alpha_{1}+\alpha_{2}}{2}-\frac{3}{2}}
\end{array}\right)} .
\end{aligned}
$$




\section{Estimation de $\left(I_{5}\right)$.}

En séparant les cas $\left|\zeta_{i}\right| \lesssim\left|\zeta_{i}-z_{i}\right|$ et $\left|\zeta_{i}-z_{i}\right| \lesssim\left|\zeta_{i}\right|,\left(I_{5}\right)$ est inférieur ou égal à une somme de termes de type:

$$
\left(I_{5}\right) \lesssim \int_{D^{\emptyset}} \frac{d v}{\left(\begin{array}{c}
\left|\zeta_{1}-z_{1}\right|\left|\zeta_{2} \zeta_{3} \zeta_{4}\right|(-\rho(\zeta)-\rho(z) \\
+|\zeta-z|^{2}+\left|\zeta_{2}\right|^{2}+\left|\zeta_{3}\right|^{2}+\left|\zeta_{4}\right|^{2} \\
+|\operatorname{Im}\langle h(\zeta, z), \zeta-z\rangle|)^{n+|\Gamma|-\alpha+\frac{\beta_{1}+\beta_{2}}{2}-1}
\end{array}\right)}
$$

posons le changement de variables:

$$
\begin{cases}t_{1}+i t_{2}=\zeta_{1}-z_{1} & \\ t_{2 j-1}+i t_{2 j}=\zeta_{j}, & \forall j \in\{2, \ldots, 4\} \\ t_{2 j-1}+i t_{2 j}=\zeta_{l}-z_{l}, & \forall l \in\{5, \ldots, n-1\} \\ t_{2 n-1}+i t_{2 n}=\rho(\zeta)+i \operatorname{Im}\langle h(\zeta, z), \zeta-z\rangle, & \end{cases}
$$

nous avons:

$$
\begin{gathered}
\left(I_{5}\right) \lesssim \int_{B_{2 n}(0, R)} \frac{d t_{1} \ldots d t_{2 n}}{\left(\left|t_{1}\right|^{2}+\left|t_{2}\right|^{2}\right)^{\frac{1}{2}} \prod_{j=2}^{4}\left(\left|t_{2 j-1}\right|^{2}+\left|t_{2 j}\right|^{2}\right)^{\frac{1}{2}}} \\
\times \frac{1}{\left(-\rho(z)+\sum_{j=1}^{n-1}\left(\left|t_{2 j-1}\right|^{2}+\left|t_{2 j}\right|^{2}\right)+\left(\left|t_{2 n-1}\right|^{2}+\left|t_{2 n}\right|^{2}\right)^{\frac{1}{2}}\right)^{n+|\Gamma|-\alpha+\frac{\beta_{1}+\beta_{2}}{2}-1}},
\end{gathered}
$$

après intégration en coordonnées polaires, $\left(I_{5}\right) \lesssim|\rho(z)|^{|\Gamma|-\left(\alpha-\frac{\beta_{1}+\beta_{2}}{2}\right)}$ si $|\Gamma|>\left(\alpha-\frac{\beta_{1}+\beta_{2}}{2}\right)$, ce qui donne le résultat d'après le lemme de HardyLittlewood.

\section{Estimation de $\left(I_{6}\right)$.}

Avec la même observation que pour $\left(I_{5}\right),\left(I_{6}\right)$ est inférieur ou égal à une somme de termes du type:

$$
\left(I_{6}\right) \lesssim \int_{D^{(4)} \cap\left\{\zeta_{4}=0\right\}} \frac{d v}{\left(\begin{array}{c}
\left|\zeta_{1}-z_{1}\right|\left|\zeta_{2} \zeta_{3}\right|(-\rho(\zeta)-\rho(z) \\
+|\zeta-z|^{2}+\left|\zeta_{2}\right|^{2}+\left|\zeta_{3}\right|^{2} \\
+|\operatorname{Im}\langle h(\zeta, z), \zeta-z\rangle|)^{n+|\Gamma|-\alpha+\frac{\alpha_{1}+\alpha_{2}}{2}-\frac{3}{2}}
\end{array}\right)}
$$


posons le changement de variables:

$$
\begin{cases}t_{1}+i t_{2}=\zeta_{1}-z_{1} & \\ t_{2 j-1}+i t_{2 j}=\zeta_{j}, & \forall j \in\{2,3\} \\ t_{l}+i t_{l+1}=\zeta_{l}-z_{l}, & \forall l \in\{4, \ldots, n-2\} \\ t_{2 n-3}+i t_{2 n-2}=\rho(\zeta)+i \operatorname{Im}\langle h(\zeta, z), \zeta-z\rangle, & \end{cases}
$$

nous avons:

$$
\begin{gathered}
\left(I_{6}\right) \lesssim \int_{B_{2 n-2}(0, R)} \frac{d t_{1} \ldots d t_{2 n-2}}{\left(\left|t_{1}\right|^{2}+\left|t_{2}\right|^{2}\right)^{\frac{1}{2}} \prod_{j=2}^{3}\left(\left|t_{2 j-1}\right|^{2}+\left|t_{2 j}\right|^{2}\right)^{\frac{1}{2}}} \\
\times \frac{1}{\left(-\rho(z)+\sum_{j=1}^{n-2}\left(\left|t_{2 j-1}\right|^{2}+\left|t_{2 j}\right|^{2}\right)+\left(\left|t_{2 n-3}\right|^{2}+\left|t_{2 n-2}\right|^{2}\right)^{\frac{1}{2}}\right)^{n+|\Gamma|-\alpha+\frac{\alpha_{1}+\alpha_{2}}{2}-\frac{3}{2}}},
\end{gathered}
$$

après intégration en coordonnées polaires, $\left(I_{6}\right) \lesssim|\rho(z)| \Gamma \mid-\left(\alpha-\frac{\alpha_{1}+\alpha_{2}}{2}\right)$ si $|\Gamma|>\left(\alpha-\frac{\alpha_{1}+\alpha_{2}}{2}\right)$, ce qui achève la preuve de la partie principale de la Proposition 2.3.

Il reste à indiquer comment l'on obtient i) et ii):

- Pour i), il suffit de remplacer dans l'expression de $T_{i}(g) g(\zeta)$ par $g(\zeta) z_{1}^{\beta_{i, 1}} \times \cdots \times z_{n}^{\beta_{i, n}}$, puis de remarquer, en utilisant la formule de Taylor dans le cas algébrique:

$$
z_{1}^{\beta_{i, 1}} \times \cdots \times z_{n}^{\beta_{i, n}}=\sum_{\substack{\left(\alpha_{1}, \ldots, \alpha_{n}\right) \\ \alpha_{j} \leq \beta_{i, j}}} \zeta_{1}^{\alpha_{1}} \times \cdots \times \zeta_{n}^{\alpha_{n}} f(\zeta, z)
$$

avec $f(\zeta, z)=\mathcal{O}\left(|\zeta-z|^{\sum_{j} \beta_{i, j}-\alpha_{j}}\right)$.

- Pour ii), il suffit de remplacer dans l'expression de $T_{i}(g), g(\zeta)$ par $z_{1}^{\theta_{1}} \times \cdots \times z_{n}^{\theta_{n}} g(\zeta)$, puis d'écrire à l'aide de la formule de Taylor, comme pour i):

$$
z_{1}^{\theta_{1}} \times \cdots \times z_{n}^{\theta_{n}}=\sum_{\substack{\left(\alpha_{1}, \ldots, \alpha_{n}\right) \\ \alpha_{j} \leq \theta_{j}}} \zeta_{1}^{\alpha_{1}} \times \cdots \times \zeta_{n}^{\alpha_{n}} f(\zeta, z)
$$

avec $f(\zeta, z)=\mathcal{O}\left(|\zeta-z|^{\sum_{j} \theta_{j}-\alpha_{j}}\right)$; enfin, il faut utiliser que $\zeta_{1}^{\alpha_{1}} \times$ $\cdots \times \zeta_{n}^{\alpha_{n}} g(\zeta) \in \Lambda_{\alpha+\frac{1}{2}} \sum_{j} \alpha_{j}(D)$. 


\section{Preuve de la Proposition 2.4}

Remarques. 1) On peut choisir $f$ définissant $V$ telle que la matrice $M=\left(\alpha_{i, j}\right)$ n'ait que des termes valant 1 ou 0 . Pour simplifier les notations, nous allons supposer que $V=f^{-1}(0)$ avec $f=\left(f_{1}, f_{2}\right)$ où

$$
\left\{\begin{array}{l}
f_{1}(z)=z_{1} z_{2} \\
f_{2}(z)=z_{3} z_{4}
\end{array}\right.
$$

le cas général s'obtient similairement.

2) Fixons $N^{\prime}>0$, d'après le Théorème 3.3 , l'opérateur $E^{N^{\prime}}$ défini par

$$
E^{N^{\prime}} g(z):=C_{n}\left\langle g(\zeta) \bar{\partial}\left[\frac{1}{f}\right]_{\{1,2\}},(b(\zeta, z))_{\{1,2\}} \wedge A^{N^{\prime}, n-2}(\zeta, z)\right\rangle,
$$

pour tout $g \in L^{\infty}(V \cap D) \cap \operatorname{Hol}(V \cap D)$ et pour tout $z \in D$, est un opérateur linéaire d'extension. De plus, il est très facile de voir que $E^{N^{\prime}} g(z)$ à une croissance "polynômiale" quand $z$ près du bord:

$$
E^{N^{\prime}} g(z)=\mathbf{O}\left(|\rho(z)|^{N}\right)
$$

si $N$ est suffisamment grand.

3) On peut remarquer également que $E^{N^{\prime}} g(z)\left(\forall N^{\prime}>0\right)$ défini une fonction continue sur $\partial D \backslash V$ car

$$
\left(-\rho(\zeta)-\rho(z)+|\zeta-z|^{2}\right) \lesssim|\rho(\zeta)+\langle h(\zeta, z), \zeta-z\rangle| .
$$

Nous avons besoin du lemme suivant:

Lemme 4.1. Soit $f=\left(f_{1}, \ldots, f_{p}\right)$ définissant $V$ comme dans le 1) de la remarque et $g \in L^{\infty}(V \cap D) \cap \operatorname{Hol}(V \cap D)$, alors pour tout $z \in \partial D \backslash V$, nous avons:

$$
\begin{aligned}
& E^{N} g(z):=\sum_{i=1}^{p} C_{i} f_{i}(z) \\
& \times\langle g(\zeta) \bar{\partial}\left[\frac{1}{f}\right]_{\{1, \ldots, p\}},(b(\zeta, z)) \overbrace{\left\{1, \ldots, i_{,} \ldots, p\right\}} \wedge \frac{\sum h_{j}(z, \zeta) d \zeta_{j}}{\langle h(z, \zeta), \zeta-z\rangle} \wedge A^{N, n-2}(\zeta, z)\rangle,
\end{aligned}
$$

pour tout $N$ assez grand.

Preuve: Il est suffisant de montrer le lemme avec $f=\left(f_{1}, f_{2}\right)$; prenons $\chi_{\varepsilon}$ une fonction $C^{\infty}(\mathbb{R})$ valant 0 si $x \geq \varepsilon$ et 1 si $x \leq \frac{\varepsilon}{2}$, écrivons la formule de représentation Berndtsson-Andersson-passare (voir [2] et [14]) en termes 
de courants, avec les paramétres ci-dessous (on utilise les notations de $[2])$ :

$$
\left\{\begin{array}{l}
s(\zeta, z):=-\rho(z) \sum_{j=1}^{n}\left(\bar{\zeta}_{j}-\bar{z}_{j}\right) d \zeta_{j}+\overline{\langle h(\zeta, z), \zeta-z\rangle} \sum_{j=1}^{n} h_{j}(\zeta, z) d \zeta_{j} \\
Q(\zeta, z):=\tilde{h}(\zeta, z)=\frac{1}{\rho(\zeta)} \sum_{j=1}^{n} h_{j}(\zeta, z) d \zeta_{j}
\end{array}\right.
$$

et $N$ un entier naturel vérifiant $E^{N^{\prime}} g(z)=\mathbf{O}\left(|\rho(z)|^{N}\right)$, pour la fonction $\chi_{\varepsilon}\left(|f|^{2}\right) E^{N^{\prime}} g$, où $|f|^{2}:=\left|f_{1}\right|^{2}+\left|f_{2}\right|^{2}$; nous obtenons, $\forall z \in D$,

$$
\begin{aligned}
& \chi_{\varepsilon}\left(|f|^{2}\right) E^{N^{\prime}} g(z) \\
& =\sum_{k=0}^{1} \sum_{|I|=2-k,|J|=k} C_{n, k}[f(z)]_{I}\left\langle\chi_{\varepsilon}\left(|f|^{2}\right) E^{N^{\prime}} g(\zeta)\left[\frac{1}{f}\right]_{I} \bar{\partial}\left[\frac{1}{f}\right]_{J},\right. \\
& \quad+C_{n}\left\langle\chi_{\varepsilon}\left(|f|^{2}\right) E^{N^{\prime}} g(\zeta) \bar{\partial}\left[\frac{1}{f}\right]_{\{1,2\}},\right. \\
& +\sum_{k=0|I|=2-k,|J|=k} D_{J, k}[f(z)]_{I}\left\langle\bar{\partial}\left(\chi_{\varepsilon}\left(|f|^{2}\right)\right) E^{N^{\prime}} g(\zeta)\left[\frac{1}{f}\right]_{I} \bar{\partial}\left[\frac{1}{f}\right]_{J}\right. \\
& \left.\quad(b(\zeta, z))_{\{1,2\}} \wedge A^{N, n-2}(\zeta, z)\right\rangle \\
& +D_{n}\left\langle\bar{\partial}\left(\chi_{\varepsilon}\left(|f|^{2}\right)\right) E^{N^{\prime}} g(\zeta) \bar{\partial}\left[\frac{1}{f}\right]_{\{1,2\}},\right. \\
& \left.(b(\zeta, z))_{\{1,2\}} \wedge B^{N, n-k}(\zeta, z) B^{N, n-2}(\zeta, z)\right\rangle
\end{aligned}
$$

avec

$$
B^{N, n-k}(\zeta, z):=\sum_{k^{\prime}=0}^{n-k-1} \frac{s \wedge(\bar{\partial} s)^{k^{\prime}-1}}{\langle s(\zeta, z), \zeta-z)^{k^{\prime}}} \wedge A^{N, n-k^{\prime}}(\zeta, z)
$$


Le courant $\bar{\partial}\left[\frac{1}{f}\right]$ est à support dans $V$, ce qui entraîne:

$$
\left\{\begin{array}{l}
\left\langle\bar{\partial}\left(\chi_{\varepsilon}\left(|f|^{2}\right)\right) E^{N^{\prime}} g(\zeta) \bar{\partial}\left[\frac{1}{f}\right]_{\{1,2\}},(b(\zeta, z))_{\{1,2\}} \wedge B^{N, n-2}(\zeta, z)\right\rangle=0 \\
\left\langle\chi_{\varepsilon}\left(|f|^{2}\right) E^{N^{\prime}} g(\zeta) \bar{\partial}\left[\frac{1}{f}\right]_{\{1,2\}},(b(\zeta, z))_{\{1,2\}} \wedge A^{N, n-2}(\zeta, z)\right\rangle=E^{N} g(z)
\end{array}\right.
$$

fixons $z_{0} \in \partial D \backslash V$, en remarquant que $\chi_{\varepsilon}\left(|f|^{2}\right)$ et $\bar{\partial}\left(\chi_{\varepsilon}\left(|f|^{2}\right)\right)$ sont nuls si $z$ est loin de $V$ et par conséquent que les intégrales ne portent que sur un petit voisinage de $V$, nous pouvons faire tendre $z$ vers $z_{0}$ dans l'égalité et obtenir, pour tout $\varepsilon$ assez petit:

$$
\begin{array}{r}
-E^{N} g\left(z_{0}\right) \\
=\sum_{k=0}^{1} \sum_{|I|=2-k,|J|=k} C_{n, k}\left[f\left(z_{0}\right)\right]_{I}\left\langle\chi_{\varepsilon}\left(|f|^{2}\right) E^{N^{\prime}} g(\zeta)\left[\frac{1}{f}\right]_{I} \bar{\partial}\left[\frac{1}{f}\right]_{J},\right. \\
\left.\left(b\left(\zeta, z_{0}\right)\right)_{J} \wedge A^{N, n-k}\left(\zeta, z_{0}\right)\right\rangle \\
+\sum_{k=0}^{1} \sum_{|I|=2-k,|J|=k} D_{n, k}\left[f\left(z_{0}\right)\right]_{I}\left\langle\bar{\partial}\left(\chi_{\varepsilon}\left(|f|^{2}\right)\right) E^{N^{\prime}} g(\zeta)\left[\frac{1}{f}\right]_{I} \bar{\partial}\left[\frac{1}{f}\right]_{J}\right. \\
\left.\left(b\left(\zeta, z_{0}\right)\right)_{J} \wedge B^{N, n-k}\left(\zeta, z_{0}\right)\right\rangle .
\end{array}
$$

Maintenant comme $z_{0} \in \partial D \backslash V$, une simple application du théorème de Lebesgue assure que

$$
\lim _{\varepsilon \rightarrow 0}\left\langle\chi_{\varepsilon}\left(|f|^{2}\right) E^{N^{\prime}} g(\zeta)\left[\frac{1}{f}\right]_{I} \bar{\partial}\left[\frac{1}{f}\right]_{J},\left(b\left(\zeta, z_{0}\right)\right)_{J} \wedge A^{N, n-k}\left(\zeta, z_{0}\right)\right\rangle=0
$$

les termes contenant $\bar{\partial}\left(\chi_{\varepsilon}\left(|f|^{2}\right)\right)$ sont plus délicats au passage à la limite, considérons par exemple le terme

$$
f_{1}(z) f_{2}(z)\left\langle\bar{\partial}\left(\chi_{\varepsilon}\left(|f|^{2}\right)\right) E^{N^{\prime}} g(\zeta)\left[\frac{1}{f}\right]_{\{1,2\}}, B^{N, n}\left(\zeta, z_{0}\right)\right\rangle:
$$


appliquons la formule de Stokes, nous avons

$$
\begin{aligned}
& f_{1}(z) f_{2}(z)\left\langle\bar{\partial}\left(\chi_{\varepsilon}\left(|f|^{2}\right)\right) E^{N^{\prime}} g(\zeta)\left[\frac{1}{f}\right]_{\{1,2\}}, B^{N, n}\left(\zeta, z_{0}\right)\right\rangle \\
= & -f_{1}(z) f_{2}(z)\left\langle\chi_{\varepsilon}\left(|f|^{2}\right) E^{N^{\prime}} g(\zeta)\left[\frac{1}{f}\right]_{\{2\}} \bar{\partial}\left[\frac{1}{f}\right]_{\{1\}}, B^{N, n}\left(\zeta, z_{0}\right)\right\rangle \\
- & f_{1}(z) f_{2}(z)\left\langle\chi_{\varepsilon}\left(|f|^{2}\right) E^{N^{\prime}} g(\zeta)\left[\frac{1}{f}\right]_{\{1\}} \bar{\partial}\left[\frac{1}{f}\right]_{\{2\}}, B^{N, n}\left(\zeta, z_{0}\right)\right\rangle \\
- & f_{1}(z) f_{2}(z)\left\langle\chi_{\varepsilon}\left(|f|^{2}\right) E^{N^{\prime}} g(\zeta)\left[\frac{1}{f}\right]_{\{1,2\}}, \bar{\partial}\left(B^{N, n}\left(\zeta, z_{0}\right)\right)\right\rangle,
\end{aligned}
$$

en utilisant le théorème de Lebesgue et en passant à la limite quant $\varepsilon$ tend vers zéro,

$$
\lim _{\varepsilon \rightarrow 0} f_{1}(z) f_{2}(z)\left\langle\bar{\partial}\left(\chi_{\varepsilon}\left(|f|^{2}\right)\right) E^{N^{\prime}} g(\zeta)\left[\frac{1}{f}\right]_{\{1,2\}}, B^{N, n}\left(\zeta, z_{0}\right)\right\rangle=0
$$

Par le même procédé sur les termes

$$
\left\{\begin{array}{l}
f_{1}(z)\left\langle\bar{\partial}\left(\chi_{\varepsilon}\left(|f|^{2}\right)\right) E^{N^{\prime}} g(\zeta)\left[\frac{1}{f}\right]_{\{1\}} \bar{\partial}\left[\frac{1}{f}\right]_{\{2\}},(b(\zeta, z))_{\{2\}} B^{N, n-1}\left(\zeta, z_{0}\right)\right\rangle \\
f_{2}(z)\left\langle\bar{\partial}\left(\chi_{\varepsilon}\left(|f|^{2}\right)\right) E^{N^{\prime}} g(\zeta)\left[\frac{1}{f}\right]_{\{2\}} \bar{\partial}\left[\frac{1}{f}\right]_{\{1\}},(b(\zeta, z))_{\{1\}} B^{N, n-1}\left(\zeta, z_{0}\right)\right\rangle,
\end{array}\right.
$$

on obtient le Lemme 4.1.

Preuve de la Proposition 2.4: D'après le Lemme 4.1, il suffit demontrer que $\forall i \in\{1,2\}$ :

$$
\begin{aligned}
\mid f_{i}(z)\left\langle g(\zeta) \bar{\partial}\left[\frac{1}{f}\right]_{\{1,2\}},\right. & (b(\zeta, z))_{\{1, \ldots, \widehat{i}, \ldots, 2\}} \\
& \left.\wedge \frac{\sum h_{j}(z, \zeta) d \zeta_{j}}{\langle h(z, \zeta), \zeta-z\rangle} \wedge A^{N, n-2}(\zeta, z)\right\rangle \mid \lesssim M
\end{aligned}
$$


$\forall z \in \partial D \backslash V$; prenons $i=1$, d'après la partie 3 ,

$$
\begin{aligned}
f_{1}(z)\left\langle g(\zeta) \bar{\partial}\left[\frac{1}{f}\right]_{\{1,2\}},(b(\zeta, z))_{\{2\}} \wedge\right. & \left.\frac{\sum h_{j}(z, \zeta) d \zeta_{j}}{\langle h(z, \zeta), \zeta-z\rangle} \wedge A^{N, n-2}(\zeta, z)\right\rangle \\
=f_{1}(z) \int_{D \cap\left\{\zeta_{1}=\zeta_{3}=0\right\}} g(\zeta) \frac{1}{\zeta_{2} \zeta_{4}}(b(\zeta, z))_{\{2\}} & \wedge \frac{\sum h_{j}(z, \zeta) d \zeta_{j}}{\langle h(z, \zeta), \zeta-z\rangle} \\
& \wedge A^{N, n-2}(\zeta, z)\left(I_{7}\right) \\
& \wedge A^{N, n-2}(\zeta, z)\left(I_{8}\right) \\
=f_{1}(z) \int_{D \cap\left\{\zeta_{1}=\zeta_{4}=0\right\}} g(\zeta) \frac{1}{\zeta_{2} \zeta_{3}}(b(\zeta, z))_{\{2\}} & \wedge \frac{\sum h_{j}(z, \zeta) d \zeta_{j}}{\langle h(z, \zeta), \zeta-z\rangle} \\
=f_{1}(z) \int_{D \cap\left\{\zeta_{2}=\zeta_{3}=0\right\}} g(\zeta) \frac{1}{\zeta_{1} \zeta_{4}}(b(\zeta, z))_{\{2\}} & \wedge \frac{\sum h_{j}(z, \zeta) d \zeta_{j}}{\langle h(z, \zeta), \zeta-z\rangle} \\
& \wedge A^{N, n-2}(\zeta, z)\left(I_{9}\right) \\
=f_{1}(z) \int_{D \cap\left\{\zeta_{2}=\zeta_{4}=0\right\}} g(\zeta) \frac{1}{\zeta_{1} \zeta_{3}}(b(\zeta, z))_{\{2\}} & \wedge \frac{\sum h_{j}(z, \zeta) d \zeta_{j}}{\langle h(z, \zeta), \zeta-z\rangle} \\
& \wedge A^{N, n-2}(\zeta, z)\left(I_{10}\right) .
\end{aligned}
$$

Il est clair qu'il suffit d'envisager le terme $\left(I_{7}\right)$; pour construire $(b(\zeta, z))_{\{2\}}$ on utilise, comme dans la partie 3, la formule de Taylor $(b(\zeta, z))_{\{2\}}=$ $\zeta_{3} d \zeta_{4}+\zeta_{4} d \zeta_{3}+\left(z_{4}-\zeta_{4}\right) d \zeta_{3}$, d'où

$$
\begin{aligned}
\left(I_{7}\right)=f_{1}(z) \int_{D \cap\left\{\zeta_{1}=\zeta_{3}=0\right\}} g(\zeta) \frac{1}{\zeta_{2}}(b(\zeta, z))_{\{2\}} & \wedge \frac{\sum h_{j}(z, \zeta) d \zeta_{j}}{\langle h(z, \zeta), \zeta-z\rangle} \\
& \wedge A^{N, n-2}(\zeta, z)\left(I_{11}\right) \\
+f_{1}(z) \int_{D \cap\left\{\zeta_{1}=\zeta_{3}=0\right\}} g(\zeta) \frac{\mathbf{O}(|\zeta-z|)}{\zeta_{2} \zeta_{4}}(b(\zeta, z))_{\{2\}} & \wedge \frac{\sum h_{j}(z, \zeta) d \zeta_{j}}{\langle h(z, \zeta), \zeta-z\rangle} \\
& \wedge A^{N, n-2}(\zeta, z)\left(I_{12}\right) .
\end{aligned}
$$




\section{Estimation de $\left(I_{11}\right)$.}

Remarquons:

$$
\begin{aligned}
A^{N, n-2}(\zeta, z)= & \frac{(\rho(\zeta))^{N+n-2}}{(\rho(\zeta)+\langle h(\zeta, z), \zeta-z\rangle)^{N+n-2}}(\bar{\partial} \tilde{h}(\zeta, z))^{n-2} \\
= & \frac{(\rho(\zeta))^{N+n-2}}{(\rho(\zeta)+\langle h(\zeta, z), \zeta-z\rangle)^{N+n-2}} \\
& \times\left(\frac{1}{\rho^{2}} \bar{\partial} \rho(\zeta) \wedge \sum h_{i}(\zeta, z) d \zeta_{i}+\frac{1}{\rho} \sum \bar{\partial} h_{i}(\zeta, z) \wedge d \zeta_{i}\right)
\end{aligned}
$$

Le morceau le plus délicat est le terme en $\frac{1}{\rho^{n-1}} \bar{\partial} \rho(\zeta) \wedge \sum h_{i}(\zeta, z) d \zeta_{i}$, mais $\sum h_{i}(z, \zeta) d \zeta_{i} \wedge \sum h_{i}(\zeta, z) d \zeta_{i}=\mathbf{O}(|\zeta-z|)$, ce qui implique:

$$
\begin{aligned}
& \left(I_{11}\right) \lesssim\left|z_{1} z_{2}\right| \int_{D \cap\left\{\zeta_{1}=\zeta_{3}=0\right\}}|g(\zeta)| \\
& \quad \times \frac{1}{\left|\zeta_{2}\right|} \frac{\mathbf{O}(|\zeta-z|)}{\left(-\rho(\zeta)+|\zeta-z|^{2}+|\operatorname{Im}\langle h(\zeta, z), \zeta-z\rangle|\right)^{n}} \\
& \lesssim\left|z_{1}\right| \int_{D \cap\left\{\zeta_{1}=\zeta_{3}=0\right\}}|g(\zeta)| \\
& \quad \times \frac{\mathbf{O}(|\zeta-z|)}{\left(-\rho(\zeta)+\left|z_{1}\right|^{2}+|\zeta-z|^{2}+|\operatorname{Im}\langle h(\zeta, z), \zeta-z\rangle|\right)^{n}} \\
& +\left|z_{1}\right| \int_{D \cap\left\{\zeta_{1}=\zeta_{3}=0\right\}}|g(\zeta)| \\
& \quad \times \frac{1}{\left|\zeta_{2}\right|} \frac{\mathbf{O}\left(|\zeta-z|^{2}\right)}{\left(-\rho(\zeta)+\left|z_{1}\right|^{2}+|\zeta-z|^{2}+|\operatorname{Im}\langle h(\zeta, z), \zeta-z\rangle|\right)^{n}}
\end{aligned}
$$

Comme à la partie 3, il suffit d'intégrer sur une boule de centre $q \in$ $\partial D \cap\left\{\zeta_{1}=\zeta_{3}=0\right\}$ et de rayon $r$ assez petit, de facon à ce que $\left|\frac{\partial \rho}{\partial \zeta_{n}}(\zeta)\right| \geq$ 
$c>0$, et de majorer $\left(I_{11}\right)$, pour $z \in \partial D \cap B\left(q, \frac{r}{2}\right) \backslash V$, par

$$
\begin{aligned}
& \left|z_{1}\right| \int_{D \cap B\left(q, \frac{r}{2}\right) \cap\left\{\zeta_{1}=\zeta_{3}=0\right\}}|g(\zeta)| \\
& \quad \times \frac{d \lambda}{\left(-\rho(\zeta)+\left|z_{1}\right|^{2}+|\zeta-z|^{2}+|\operatorname{Im}\langle h(\zeta, z), \zeta-z\rangle|\right)^{n-\frac{1}{2}}} \\
& +\left|z_{1}\right| \int_{D \cap B\left(q, \frac{r}{2}\right) \cap\left\{\zeta_{1}=\zeta_{3}=0\right\}}|g(\zeta)| \\
& \quad \times \frac{1}{\left|\zeta_{2}\right|} \frac{d \lambda}{\left(-\rho(\zeta)+\left|z_{1}\right|^{2}+|\zeta-z|^{2}+|\operatorname{Im}\langle h(\zeta, z), \zeta-z\rangle|\right)^{n-1}}
\end{aligned}
$$

l'estimation s'obtient en faisant le même type de changement de variables que pour la Proposition 2.3; l'estimation de $\left(I_{12}\right)$ est similaire à celle de $\left(I_{11}\right)$, ce qui achève la preuve de la Proposition 2.4.

\section{Remarque finale}

A notre avis, il serait très intéressant d'avoir l'équivalent du Théorème 1.1, dans les domaines convexes de type fini. Ceci n'est pas hors de portée, car nous pouvons construire, dans ce cas, des noyaux intégraux donnant les solutions du problème de division à l'aide de la fonction support de Diederich-Fornaess de [5]. Mais dans cette situation, le problème est plus subtil comme le prouve l'exemple suivant: prenons

$$
\begin{aligned}
& D:=\left\{\rho(z)=\left|z_{1}\right|^{2 q}+\left|z_{2}\right|^{2}+\left|z_{3}\right|^{2}+\left|z_{4}\right|^{2}-1<0\right\}, \\
& f=\left(f_{1}, f_{2}\right)=\left(z_{1} z_{2}, z_{1}^{n}+z_{3}\right)
\end{aligned}
$$

avec $n>1$ et $n \ll q$. Il est clair que $f$ est une intersection complète à croisements normaux et que $g \in \operatorname{Hol}(D)$ s'écrit $z_{1} z_{2} g_{1}(z)+\left(z_{1}^{n}+z_{3}\right) g_{2}(z)$ si et seulement si $g$ s'annule sur $f^{-1}(0)$ (dans la suite on notera cette condition $(* *))$. Considérons $g(z)=\frac{z_{2} z_{3}}{1-z_{4}}, g$ est bornée et vérifie $(* *)$. On a une décomposition immédiate:

$g(z)=z_{1} z_{2}\left(\frac{z_{1}^{n-1}}{1-z_{4}}\right)-\left(z_{1}^{n}+z_{3}\right)\left(\frac{z_{2}}{1-z_{4}}\right)$ avec $\frac{z_{1}^{n-1}}{1-z_{4}} \in B_{\frac{n-1}{2 q}-1}(D)$. Supposons qu'il existe $g_{1}, g_{2}$ vérifiant $z_{1} z_{2} g_{1}(z)+\left(z_{1}^{n}+z_{3}\right) g_{2}(z)$ avec $g_{1} \in B_{k}(D)$ et $k>\frac{n-1}{2 q}-1$, alors $\left.\frac{z_{1}^{n-1}}{1-z_{4}}\right|_{z_{1}^{n}+z_{3}=0}$ aurait une extension holomorphe à $D$ dans un meilleur espace que $B_{\frac{n-1}{2 q}-1}(D)$, ce qui est impossible (pour le voir, il suffit d'adapter la preuve dans [11]). Si le Théorème 1.1 s'étendait "in extenso", $g$ étant très régulière sauf en 
$(0,0,0,1)$ et de plus, en ce point, $z_{1}$ étant une direction de faible pseudoconvexité et $z_{2}$ de stricte pseudoconvexité, on pourrait s'attendre à trouver $g_{1}$ dans $B_{-\frac{1}{2}-\frac{1}{2 q}}(D)$. Ceci montre que dans les domaines faiblement pseudoconvexes, même dans le cas des intersections complètes à croisements normaux, on ne peut pas toujours trouver une décomposition sans compensations entre les différents termes.

\section{Références}

[1] B. Berndtsson, The extension theorem of Ohsawa-Takegoshi and the theorem of Donnelly-Fefferman, Ann. Inst. Fourier (Grenoble) 46(4) (1996), 1083-1094.

[2] B. Berndtsson et M. Andersson, Henkin-Ramirez formulas with weight factors, Ann. Inst. Fourier (Grenoble) 32(3) (1982), 91-110.

[3] P. Bonneau, A. Cumenge et A. ZÉriahi, Division dans les espaces de Lipschitz de fonctions holomorphes, in "Séminaire d'analyse P. Lelong-P. Dolbeault-H. Skoda, années 1983/1984", Lecture Notes in Math. 1198, Springer, Berlin, 1986, pp. 73-87.

[4] N. R. ColefF ET M. E. Herrera, "Les courants résiduels associés à une forme méromorphe", Lecture Notes in Math. 633, Springer, Berlin, 1978.

[5] K. Diederich et J. E. Fornæss, Support functions for convex domains of finite type, Math. Z. 230(1) (1999), 145-164.

[6] K. Diederich ET E. MAzzilli, A remark on the theorem of Ohsawa-Takegoshi, Nagoya Math. J. 158 (2000), 185-189.

[7] P. Dolbeault, Sur la structure des courants résiduels, Rev. Roumaine Math. Pures Appl. 33(1-2) (1988), 31-37.

[8] P. Dolbeault, Sur la théorie des résidus en plusieurs variables, Astérisque 217 (1993), 85-101.

[9] P. Dolbeault, Résidus et courants, in "Questions on Algebraic Varieties" (C.I.M.E., III Ciclo, Varenna, 1969), Edizioni Cremonese, Rome, 1970, pp. 1-28.

[10] J. E. FornaEss, Embedding strictly pseudoconvex domains in convex domains, Amer. J. Math. 98(2) (1976), 529-569.

[11] E. Mazzilli, Extension des fonctions holomorphes, C. R. Acad. Sci. Paris Sér. I Math. 321(7) (1995), 831-836.

[12] J. M. Ortega et J. FÀbregA, Division and extension in weighted Bergman-Sobolev spaces, Publ. Mat. 36(2B) (1992), 837-859 (1993). 
[13] M. PAssare, A new division formula for complete intersections, in: "Proceedings of the Tenth Conference on Analytic Functions" (Szczyrk, 1990), vol. 55, 1991, pp. 283-286.

[14] M. PAssare, Residues, currents, and their relation to ideals of holomorphic functions, Math. Scand. 62(1) (1988), 75-152.

[15] H. SkodA, Application des techniques $L^{2}$ à la théorie des idéaux d'une algèbre de fonctions holomorphes avec poids, Ann. Sci. École Norm. Sup. (4) 5 (1972), 545-579.

Laboratoire de Géométrie, Analyse et Topologie

C.N.R.S. U.R.A. D751

U.F.R. de Mathématiques

Université Lille I

59655 Villeneuve d'Ascq Cedex

France

E-mail address: maati@gat.univ-lille1.fr

E-mail address: mazzilli@gat.univ-lille1.fr

Primera versió rebuda el 30 de juny de 2000, darrera versió rebuda el 5 de marc de 2001. 